\title{
The HeartMate 3 pump: Overcoming the hemocompatibility gap
}

Joseph C. Cleveland, Jr, MD, FACS, FACC, ${ }^{a}$ and Daniel J. Goldstein, MD, FACS, FACC ${ }^{b}$

\footnotetext{
From the ${ }^{\mathrm{a}}$ Cardiac Transplantation, Division of Cardiothoracic Surgery, University of Colorado Anschutz Medical Center, Aurora, Colo; and ${ }^{\mathrm{b} C a r d i a c}$ Transplantation and Mechanical Circulatory Support Programs, Department of Cardiothoracic Surgery, Montefiore Medical Center, Albert Einstein College of Medicine, Bronx, NY.

Disclosures: Authors both disclose grant support and travel from Abbott Medical as co-principal investigators of the MOMENTUM3 Trial.

Received for publication April 13, 2018; revisions received April 13, 2018; accepted for publication May 1, 2018; available ahead of print July 3, 2018.

Address for reprints: Joseph C. Cleveland, Jr, MD, FACS, FACC, University of Colorado Anschutz Medical Center, Academic Office One, Mail Stop C-310, 12631 E 17th Ave, Room 6602, Aurora, CO 80045 (E-mail: joseph. cleveland@ucdenver.edu).

J Thorac Cardiovasc Surg 2018;156:2140-2

$0022-5223 / \$ 36.00$

Copyright $(2018$ by The American Association for Thoracic Surgery

https://doi.org/10.1016/j.jtcvs.2018.05.055
}

\begin{abstract}
Now this is not the end. It is not even the beginning of
the end. But it is, perhaps, the end of the beginning.
\end{abstract}

-Winston Churchill

The Lord Mayor's Luncheon, November 10, 1942

The use of continuous-flow left ventricular assist systems (LVAS) has led to dramatic survival and quality of life improvements for patients with advanced heart failure refractory to medical therapy. ${ }^{1}$ These clinical outcomes have enabled more widespread applicability, driven by the engineering advances of miniaturization and fewer moving parts, which have enhanced device durability relative to earlier, larger devices with displacement chambers designed to preserve pulsatile flow. ${ }^{2}$ A spectrum of adverse events rarely seen with the more physiologic pulsatile flow pumps has been encountered, however, including pump thrombosis and mucosal bleeding, particularly in the gastrointestinal tract. Importantly, strokes, which were frequently observed with pulsatile devices, have remained a high-frequency event with continuous-flow pumps. ${ }^{3}$ These complications, which reflect the clinical interaction between the patient and the pump, are now defined as hemocompatibility-related adverse events. ${ }^{4}$

Two continuous-flow devices, the axial HeartMate II (HM II; Abbott Medical, St Paul, Minn) and the centrifugal HeartWare HVAD (Medtronic, Minneapolis, Minn) are available for use as a bridge to transplantation and for long-term use in transplant-ineligible patients. The HM II mechanical bearing axial continuous-flow blood pump is uniquely prone to pump malfunction as a result of de novo pump thrombosis, a complication encountered in $6 \%$ to $15 \%$ of patients undergoing short-term or longterm support. ${ }^{5,6}$ This complication invariably requires a second operation to exchange the pump, which remains prone to recurrent such events as well as strokes. The HVAD pump, a centrifugal flood device, is also known to

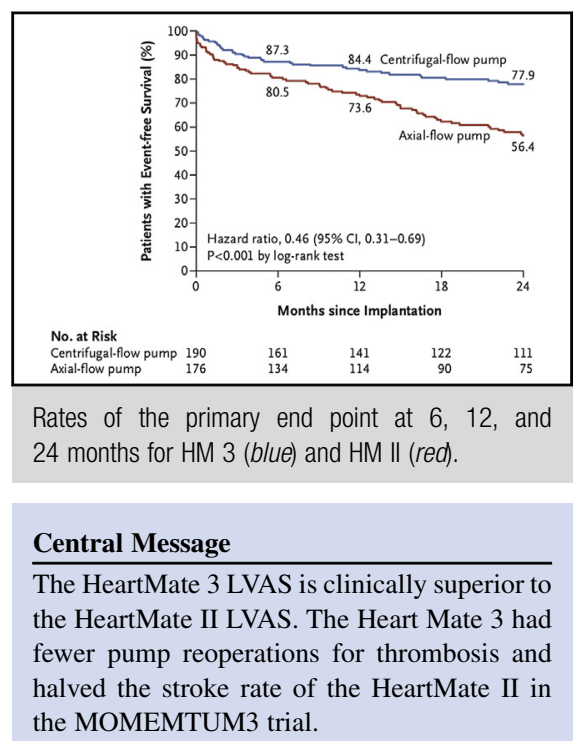

suffer from pump thrombosis, albeit at a lower rate than the HM II pump, but has also been shown to be associated with an excess rate of stroke in the long term. This excess stroke rate with the HVAD has been linked to blood pressure targets and, despite tight control of blood pressure, remains a significant risk compared with the HM II device. ${ }^{8}$ Gastrointestinal bleeding is a common adverse complication with these devices that results in frequent hospitalization, blood transfusion, alteration of antithrombotic therapy, and increased medical resource use. These complications exemplify the delicate balance between thrombosis and bleeding with left ventricular assist systems and determine the eventual clinical expression of hemocompatibility. The field thus has been in search of a pump designed to address this specific crux of clinical blight.

The Multicenter Study of MagLev Technology in Patients Undergoing Mechanical Circulatory Support Therapy with HeartMate 3 (MOMENTUM 3) Trial is a randomized controlled trial that compares the HeartMate 3 (HM 3) with the HMII in patients with advanced heart failure. ${ }^{9}$ The HM 3 is built on the framework of a fully magnetically levitated rotor pump that is centrifugal in flow paths and was engineered to avert complications of thrombosis within the pump. Wide passages for blood flow, frictionless propulsion with magnetic levitation, and an intrinsic pulse designed to reduce blood stasis were all incorporated into this novel centrifugal pump. ${ }^{9,10}$ Unlike the HM II pump, which 
requires an abdominal pocket for securing the pump, the HM 3 is placed in the thorax, in a manner similar to the HVAD pump. MOMENTUM3 was conducted in 69 experienced LVAS centers in the United States. The study is designed as an adaptive trial with several innovative features. The trial includes an all-comer population whereby patients are enrolled with single inclusion and exclusion criteria irrespective of the intended goal of the device-either bridge-to-transplant or destination therapy. The trial is set to evaluate safety after the initial random allocation of prespecified patients, followed by several stops, each powered to evaluate conclusively the primary end point. The first stop at 6 months, deemed the shortterm cohort, included 294 patients; the second analysis at 2 years was prespecified in 366 patients as the long-term end point, and a third analysis is ongoing in the full cohort of 1028 patients to validate and establish findings at previous stops. ${ }^{9}$ The primary end point of the trial is survival free of a disabling stroke or reoperation to replace or remove a malfunctioning pump.

In the short-term outcome analysis of the trial, noninferiority and even superiority of the HM 3 to the HM II on the primary end point was demonstrated in this cohort. ${ }^{10}$ This study phase showed absence of pump thrombosis, the primary driver for reduced pump reoperations, in the HM 3 arm of the trial. There was no difference in overall 6month survival or disabling stroke rates.

The MOMENTUM 3 long-term cohort data were presented and published recently. ${ }^{11}$ In this series, 366 patients (294 original patients plus an additional 72) randomly allocated (190 to the HM 3 and 176 to the HM II) were followed up for 2 years. The HM 3 again demonstrated superiority relative to the HMII pump with regard to the primary end point (Figure 1). The individual components of the primary end point suggest that whereas the major difference was a 10 -fold increase in reoperations for a malfunctioning pump in the HM II arm, overall survival also trended to favor the HM 3. Survivals at 2 years were $83 \%$ for the HM 3 versus $76 \%$ for those receiving the HMII pump. It should be noted that the prespecified cohort of 366 patients was not powered to show a difference in survival. Freedom from a disabling stroke was $93 \%$ in both the HM 3 and HM2 groups, indicating no difference in this lowfrequency event. With respect to reoperations for a malfunctioning pump, only 3 such events occurred in the HM 3 cohort-one for an electrical issue (an abrupt electrical failure caused by driveline fault), 1 for device infection, and 1 for an outflow graft twist. ${ }^{12}$ No device malfunctions intrinsic to the pump itself were noted. Of the 10-fold higher events in the HM II arm $(n=30)$, two-thirds were a result of pump thrombosis.

Two other important observations from an analysis of adverse events are germane to a focused discussion. First-time occurrence of a gastrointestinal bleeding event

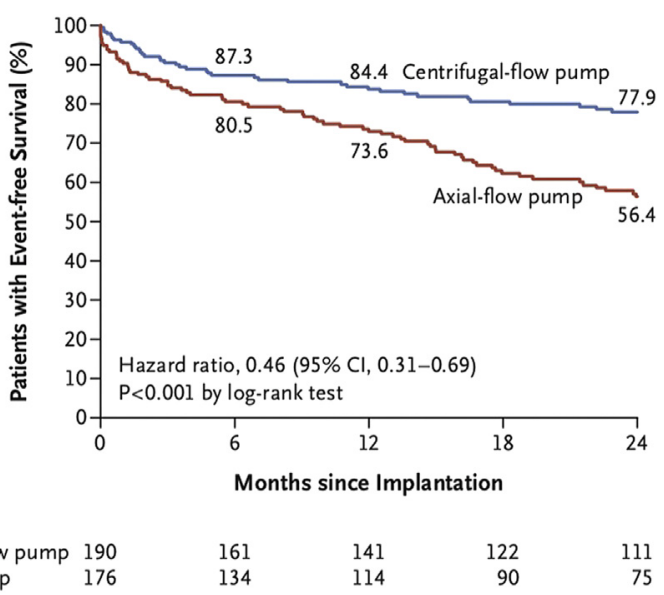

$\begin{array}{llllrr}\text { No. at Risk } & & & & & \\ \text { Centrifugal-flow pump } & 190 & 161 & 141 & 122 & 111 \\ \text { Axial-flow pump } & 176 & 134 & 114 & 90 & 75\end{array}$

FIGURE 1. Kaplan-Meier estimates of the primary end point in the intention-to-treat population. The intention-to-treat population included all patients who underwent random assignment. The primary end point was a composite of survival free of disabling stroke (with disabling stroke indicated by a modified Rankin score $>3$; scores range from $0-6$, with higher scores indicating more severe disability) and survival free of reoperation to replace or remove a malfunctioning device at 24 months after implantation. Rates of the primary end point at $0,6,12$, and 24 months are shown below the graph. $C I$, Confidence interval. From Mehra MR, Goldstein DJ, Uriel N, Cleveland JC Jr, Yuzefpolskaya M, Salerno C, et al; MOMENTUM 3 Investigators. Two-year outcomes with a magnetically levitated cardiac pump in heart failure. N Engl J Med. 2018;378:138695. Copyright (c) 2018 Massachusetts Medical Society. Reprinted with permission from Massachusetts Medical Society.

was not different between the 2 device arms; however, a numerically lower rate of recurrent gastrointestinal bleeding events was noted in the HM 3 arm. More importantly, this trial observed a marked reduction in the overall incidence of stroke with the HM 3 pump. The absolute 2-year stroke rate observed in the HM 3 arm of the long-term cohort of MOMENTUM 3 was $10 \%$ (vs $19 \%$ for the HM II pump), the lowest such rate attained in any LVAS trial. The halving of the stroke rate was similarly seen with both ischemic and hemorrhagic strokes. In aggregate, these findings point to the notion that the HM 3 pump is more hemocompatible than the control pump (HM II). ${ }^{13}$

The fully enrolled MOMENTUM3 trial includes1028 patients, who will be followed up to at least 2 years. This represents the largest trial in this space and will allow important validation of the findings observed in the current analyses. Although no individual subgroup has accounted for the superiority of the HM 3 pump, this entire cohort will yield a greater ability to look into patient characteristics, and this may inform improved selection and refinement for decision making. ${ }^{14}$ We believe that this larger experience will shed greater light on issues that could inform better functional outcomes for patients, including optimization of pump speed, better blood pressure targets, and desired antithrombotic 
strategies. The ongoing continued access protocol, which is nearing an additional 1500 enrolled patients, will provide ongoing ability to validate these outcomes and help establish decision trees to inform clinicians. One of the key issues raised by the MOMENTUM 3 trial relates to whether the anticoagulation regimens used to support the HM 3 can in fact be relaxed. A single-arm study has been completed in Europe to assess the feasibility of this approach to target a lower anticoagulation intensity with an international normalized ratio of 1.5 to $1.9{ }^{15}$ Certainly, this early experience will inform the need for a large-scale randomized study to evaluate the usefulness of low-intensity anticoagulation with the HM 3 pump.

MOMENTUM3 represents an important iterative advancement in LVAS. It is the largest comparative effectiveness trial in the field of mechanical circulatory support. The 2-year data suggest that the quest for a more hemocompatible pump has been achieved. We must not rest on this success, however; adverse events remain frequent, they are resilient and too costly, and they continue to affect the quality of life of our patients. All these factors limit more widespread use. In the future, we must search for lower anticoagulation targets, strive for a better understanding of neurologic outcomes, and address new mechanical problems that are emerging with such a technologic advance. More importantly, a fully internalized power source represents the next disruptive phase in this technologic offering, one that has the greatest opportunity to further affect the quality-adjusted life years for these patients living with advanced heart failure. At the very least, we are firmly entrenched on a new plateau of durable LVAS technology from which to anchor our future endeavors.

\section{References}

1. Slaughter MS, Rogers JG, Milano CA, Russell SD, Conte JV, Feldman D, et al; HeartMate II Investigators. Advanced heart failure treated with continuous-flow left ventricular assist device. $N$ Engl J Med. 2009;361:2241-51.
2. Kirklin JK, Naftel DC, Pagani FD, Kormos RL, Stevenson LW, Blume ED, et al. Seventh INTERMACS annual report: 15,000 patients and counting. J Heart Lung Transplant. 2015;34:1495-504.

3. Willey JZ, Gavalas MV, Trinh PN, Yuzefpolskaya M, Reshad Garan A, Levin AP, et al. Outcomes after stroke complicating left ventricular assist device. J Heart Lung Transplant. 2016;35:1003-9.

4. Mehra MR. The burden of haemocompatibility with left ventricular assist systems: a complex weave. Eur Heart J. February 23, 2017 [Epub ahead of print].

5. Stewart GC, Givertz MM, Mehra MR. Pump thrombosis redux. J Heart Lung Transplant. 2015;34:1511-4.

6. Maltais S, Kilic A, Nathan S, Keebler M, Emani S, Ransom J, et al; PREVENT Study Investigators. PREVENtion of HeartMate II pump thrombosis through clinical management: the PREVENT multi-center study. J Heart Lung Transplant. 2017;36:1-12.

7. Rogers JG, Pagani FD, Tatooles AJ, Bhat G, Slaughter MS, Birks EJ, et al. Intrapericardial left ventricular assist device for advanced hear failure. $N$ Engl J Med. 2017;376:451-60.

8. Teuteberg JJ, Slaughter MS, Rogers JG, McGee EC, Pagani FD, Gordon R, et al; ADVANCE Trial Investigators. The HVAD left ventricular assist device: risk factors for neurological events and risk mitigation strategies. JACC Heart Fail. 2015;3:818-28.

9. Heatley G, Sood P, Goldstein D, Uriel N, Cleveland J, Middlebrook D, et al; MOMENTUM 3 Investigators. Clinical trial design and rationale of the multicenter study of MagLev technology in patients undergoing mechanical circulatory support therapy with HeartMate 3 (MOMENTUM 3 ) investigational device exemption clinical study protocol. J Heart Lung Transplant. 2016;35: 528-36.

10. Mehra MR, Naka Y, Uriel N, Goldstein DJ, Cleveland JC Jr, Colombo PC, et al; MOMENTUM 3 Investigators. A fully magnetically levitated circulatory pump for advanced heart failure. $N$ Engl J Med. 2017;376:440-50.

11. Mehra MR, Goldstein DJ, Uriel N, Cleveland JC Jr, Yuzefpolskaya M, Salerno C, et al; MOMENTUM 3 Investigators. Two-year outcomes with a magnetically levitated cardiac pump in heart failure. $N$ Engl J Med. 2018; 378:1386-95.

12. Potapov EV, Netuka I, Kaufmann F, Falk V, Mehra MR. Strategy for surgical correction and mitigation of outflow graft twist with a centrifugal-flow left ventricular assist system. J Heart Lung Transplant. 2018;37:670-3.

13. Uriel N, Colombo PC, Cleveland JC Jr, Long JW, Salerno C, Goldstein DJ, et al. Hemocompatibility-related outcomes in the MOMENTUM 3 trial at 6 months: a randomized controlled study of a fully magnetically levitated pump in advanced heart failure. Circulation. 2017;135:2003-12.

14. Goldstein DJ, Mehra MR, Naka Y, Salerno C, Uriel N, Dean D, et al; MOMENTUM 3 Investigators. Impact of age, sex, therapeutic intent, race and severity of advanced heart failure on short-term principal outcomes in the MOMENTUM 3 trial. J Heart Lung Transplant. 2018;37:7-14.

15. US National Library of Medicine, ClinicalTrials.gov. MAGENTUM 1 HeartMate 3 LVAS: A Single Center Prospective Controlled Study (MAGENTUM 1). Available at: https://clinicaltrials.gov/ct2/show/NCT03078374. Accessed April $1,2018$. 